\title{
Is It Time for Genome Sequencing to Replace Exome Sequencing?
}

\section{Kanay $\mathbf{Y}^{1,2 *}$}

${ }^{1}$ Department of Medical Genetics, Acibadem Mehmet Ali Aydinlar University Medical School, Kucukbakkalkoy, Istanbul, Turkey

${ }^{2}$ Acibadem Labgen Genetic Diagnosis Center, Istanbul, Turkey

\section{Introduction}

We owe the rapid development of molecular medicine to the use of new technologies in the diagnosis. Most of all genetic diseases are single gene diseases with typical Mendelian inheritance patterns. Most of them are the result of mutations that can be detected by today's technologies: sequence changes that can be detected by sequencing methods and copy number variations (CNVs) that can be detected by array methods. Next Generation Sequencing (NGS) is a technology that allows the examination of a DNA segment that is too large to be obtained by conventional Sanger sequence analysis in a single experiment. Conventional sequence analysis was developed in 1975 by Sanger and named after the method. The NGS simply provides an output increase. Sanger sequencing is still the gold standard method, contrary to what is believed; as well as clinically the most preferred method of verification [1-3]. Today, the sanger sequencing method is used to investigate a specific region, gene, or specific mutation for "targeted sequencing", in addition to verification.

Next Generation Sequencing (NGS) targeted DNA in two different ways; partially or completely. Partial DNA sequence analysis is targeted. If we are targeting a portion of DNA with NGS, this is probably panel sequencing. For example, a commercial panel kit targeting 26 tumor suppressor genes associated with hereditary cancer predisposition is frequently used in the European market.

\section{Advantages and disadvantages of Whole Exome Sequencing (WES)}

Among targeted sequencing alternatives, the largest scope has been sequencing with exome. In other words, the most comprehensive range of targeted sequencing methods is whole exome sequencing [4].

Human Genome Project was completed in 2002. So far, we know only the coding regions of the genes that make up the difference and it accounts for only $1-2 \%$ of the genome. This reduces storage and analysis costs. Approximately $85 \%$ of pathogenic genetic variations include mutations in this coding region. In this context, compared to WGS, WES has lower cost and higher efficiency. This is why WES has been routinely used and has now enabled the discovery of many diseasecausing genes. But what about the CNVs? In 2010, chromosomal microarray was accepted as the first-tier test in the presence of unexplained developmental delay, intellectual disability (DD / ID), autism spectrum disorder (ASD), or multiple congenital anomalies (MCA). Therefore, we expect the results of chromosomal microarray to be ready before WES analysis. But this is not enough. In countries like Turkey, consanguineous marriages and rare recessive disease incidence is very high. We are detecting a heterozygous mutation in many recessive OMIM genes. In case of clinical suspicion, meaning that for the genes those are clinically relevant, for the other allele, it requires screening of intragenic deletions and duplications using a method such as qPCR or MLPA. The cost is increasing with Sanger's confirmations and family analyzes. Actually, there is a very effective way to scan such mutations in one step: HD Arrays of various manufacturers or Exon Array (Affymetrix, inc, Thermo Fisher Scientific USA). Today WES and exon array together cost around $\$ 1000$.
The diagnostic yield of WES is low, even if supplemented by the techniques for the detection of copy number variants (CNVs). This rate is so heterogeneous that in the literature, the diagnostic yield with WES may vary from 10 to 50 percent $[5,6]$.

\section{Advantages and disadvantages of Whole Genome Sequencing} (WGS)

The whole genome sequencing is the technique that allows the whole of the DNA to be genome-wide analyzed. WGS promises to reduce all conventional genetic algorithms to a single step. It is possible to detect even structural variations including balanced chromosomal translocations through WGS, which also targets all of the genome and even encompasses the entire human DNA sequence, including the copy number variants on it.

It also has some technical advantages. WGS is PCR free, and does not require an enrichment step, so it generates a uniform coverage of the genome. This provides an advantage for CNV detection. There is no limitation for sequencing read lengths with WGS. It is not a problematic issue to read repeat sequences.

The diagnostic yield of WGS is still not $100 \%$. Of course, besides technically challenging regions of high GC content, large repeats etc, the reason is not only technical. We classify the variants obtained by a knowledge that is not progressing in parallel with today's technology and try to reach a pathogenic diagnostic variant. Even the population frequencies of the variants we have identified have not been fully established. This results in a longer list of variants of unknowns (VUS) [7-11]. Obviously, as the scope of the test expands to WGS, the number of VUS will increase further.

WGS still requires several thousand dollars to be overlooked. It is a costlier approach to securely store and analyze data that is close to 100 gigabytes with WGS. For centers that have the chance to use a high-throughput platform, like HiSeq X-Ten of Illumina and others of course, costs are even lower. Probably the server capabilities of these centers have also improved [12].

\section{Conclusion}

Currently, the only reason that influences WES and WGS is the cost. WES represents the primary alternative to gene panel testing. As the difference between costs becomes narrower, the preferences will

*Corresponding author: Dr. Kanay Yararbas, Department of Medical Genetics, Acibadem Mehmet Ali Aydinlar University Medical School, Kucukbakkalkoy, Istanbul, Turkey, Tel: 90216500 4785; E-mail: kanayyararbas@gmail.com, kanay.yararbas@acibadem.edu.tr

Received November 25, 2018; Accepted December 27, 2018; Published December 31, 2018

Citation: Kanay Y (2018) Is It Time for Genome Sequencing to Replace Exome Sequencing?. J Mol Genet Med 12: 384 doi:10.4172/1747-0862.1000384

Copyright: (02018 Kanay Y. This is an open-access article distributed under the terms of the Creative Commons Attribution License, which permits unrestricted use, distribution, and reproduction in any medium, provided the original author and source are credited 
Citation: Kanay Y (2018) Is It Time for Genome Sequencing to Replace Exome Sequencing?. J Mol Genet Med 12: 384 doi:10.4172/17470862.1000384

shift to the WGS direction and the entire diagnostic analysis algorithm will fall into single step.

\section{References}

1. Sanger F, Coulson AR (1975) A rapid method for determining sequences in DNA by primed synthesis with DNA polymerase. J Mol Biol 94: 441-448.

2. Sanger F, Nicklen S, Coulson AR (1977) DNA sequencing with chainterminating inhibitors. PNAS 74: 5463-5467.

3. Choi M, Scholl UI, Ji W, Liu T, Tikhonova IR, et al. (2009) Genetic diagnosis by whole exome capture and massively parallel DNA sequencing. Proc Natl Acad Sci USA 106: 19096-19101.

4. Biesecker LG, Green RC (2014) Diagnostic clinical genome and exome sequencing. N Engl J Med 371: 1169-1170.

5. Haskell GT, Adams MC, Fan Z, Amin K, Badillo RJG, et al. (2018) Diagnostic utility of exome sequencing in the evaluation of neuromuscular disorders. Neurol Genet 4: e212.

6. Dillon OJ, Lunke S, Stark Z, Yeung A, Thorne N, et al. (2018) Exome sequencing has higher diagnostic yield compared to simulated disease-specific panels in children with suspected monogenic disorders. European J of Hum Genet 26: 644-651.

7. Miller DT, Adam MP, Aradhya S, Biesecker LG, Brothman AR, et al. (2010) Consensus statement: Chromosomal microarray is a first-tier clinical diagnostic test for individuals with developmental disabilities or congenital anomalies. Am J Hum Genet 86: 749-764.

8. Guo W, Zhu X, Yan L, Qiao J (2018) The present and future of whole-exome sequencing in studying and treating human reproductive disorders. J Genet Genom 45: 517-525.

9. Rabbani B, Tekin M, Mahdieh N (2014) The promise of whole-exome sequencing in medical genetics. J Hum Genet 59: 5-15.

10. Rabbani B, Mahdieh N, Hosomichi K, Nakaoka H, Inoue I (2012) Nextgeneration sequencing: Impact of exome sequencing in characterizing Mendelian disorders. J Hum Genet 57: 621-632.

11. Feero WG (2014) Clinical application of whole-genome sequencing proceed with care. JAMA 311: 1017-1019.

12. Bao R, Huang L, Andrade J, Tan W, Kibbe W, et al. (2014) Review of current methods, applications, and data management for the bioinformatics analysis of whole exome sequencing. Cancer Inform 13: 67-82. 